\title{
EFFECTIVENESS OF DIFFERENT ACCELERATED PARTIAL BREAST IRRADIATION TECHNIQUES FOR THE TREATMENT OF BREAST CANCER PATIENTS: SYSTEMATIC REVIEW USING INDIRECT COMPARISONS OF RANDOMIZED CLINICAL TRIALS [EFFECTIVENESS OF DIFFERENT APBI TECHNIQUES]
}

Gustavo Nader Marta*, Gustavo José Martiniano Porfirio², Ana Luiza Cabrera Martimbianco², Jessica Barrett³, José Luiz Barbosa Bevilacqua ${ }^{4}$, Philip Poortmans ${ }^{5}$, Rachel Riera ${ }^{2,6}$

\author{
1Department of Radiation Oncology, Hospital Sírio-Libanês. Department of Radiology and Oncology, Radiation Oncology \\ Unit., Universidade de São Paulo. Instituto do Câncer do Estado de São Paulo (ICESP) - Faculdade de Medicina da \\ Universidade de São Paulo - Sao Paulo (SP), Brazil. \\ ${ }^{2}$ Cochrane Brazil - Sao Paulo (SP), Brazil. \\ ${ }^{3} \mathrm{MRC}$ Biostatistics Unit, University of Cambridge - Cambridge, United Kingdom. \\ ${ }^{4}$ Department of Breast Cancer, Hospital Sírio-Libanês - Sao Paulo (SP), Brazil. \\ ${ }^{5}$ Department of Radiation Oncology - Institut Curie - Paris, France. \\ ${ }^{6}$ Discipline of Emergency Medicine and Evidence-Based Medicine, Universidade Federal de São Paulo, Escola Paulista de \\ Medicina - Sao Paulo (SP), Brazil. \\ *Corresponding author: gustavo.marta@hc.fm.usp.br
}

Background: Numerous accelerated partial breast irradiation APBI techniques are available for clinical practice. This systematic review was conducted to compare the effectiveness of different APBI techniques for the treatment of breast cancer patients. Methods: Systematic review of randomized controlled trials of APBI versus WBI. The data from APBI studies were extracted for the analyses. Indirect comparisons were used to compare different APBI techniques. Results: Ten studies fulfilled the inclusion criteria. A total of 4343 patients were included, most of them with tumor stage T1-T2 and N0. Regarding APBI techniques, six trials used external beam radiation therapy; one intraoperative electrons; one intraoperative low-energy photons; one brachytherapy; and one external beam radiation therapy or brachytherapy. The indirect comparisons related to 5-years local control and 5-years overall survival were not significantly different between APBI techniques. Conclusions: Based on indirect comparisons, no differences in clinical outcomes were observed among diverse APBI techniques in published clinical trials that formally compared WBI to APBI. However wide confidence intervals and high risk of inconsistency precluded a sound conclusion. Further head-to- head clinical trials comparing different APBI techniques are required to confirm our findings. Studies comparing different techniques using individual participant data and/or real-life data from population-based studies/registries could also provide more robust results. 